\title{
Surface Modification of Zinc Oxide by 3- aminopropyltiethoxysilane and a Comparative Study of Effect of Corrosion on Carbon Steel With Epoxy Containing Graphene Oxide-Zinc Oxide (GO-ZnO) Hybrids
}

\author{
Othman Nurul Husna, ${ }^{*}$, Mustapha Mazli ${ }^{2}$, and Che Ismail Mokhtar ${ }^{2}$ \\ ${ }^{1}$ Center for Corrosion Research, Universiti Teknologi PETRONAS \\ ${ }^{2}$ Mechanical Engineering Department, Universiti Teknologi PETRONAS
}

\begin{abstract}
The use of coupling agent, 3-aminopropyltiethoxysilane (APTES) in the silanization reaction with metal oxides plays an important role to ensure that additional chemical modification can successfully be achieved. Studies have shown that introducing metal oxides onto graphene oxide sheets can improve the dispersion of sheets in a polymeric matrix, contributing to its excellent anti-corrosion properties. Hence, two methods of APTES attachment has been explored, where the first method utilizes a reflux process to introduce siloxane bonds to the $\mathrm{ZnO} N \mathrm{NP}$ surface; the latter usesuse of ultrasonication to stimulate the functionalization of $\mathrm{ZnO}$ NPs. Fourier transform infrared spectroscopy (FT-IR), X-ray photoelectron spectroscopy (XPS) were employed to characterize the APTESFunctionalized $\mathrm{ZnO}$ and the precipitation on the surface of GO sheets. The effect of GO-ZnO produced by the different types of functionalized $\mathrm{ZnO}$ on the corrosion protection and barrier performance of epoxy coating was investigated by electrochemical impedence spectroscopy (EIS). The results revealed that the long duration of reaction time provided by the reflux method managed to increase the number of siloxane bonds on the $\mathrm{ZnO}$ surface, allowing more amine groups to be attached onto the GO sheets and thus improve the corrosion resistance of epoxy.
\end{abstract}

\section{Introduction}

Surface modification of metal oxides has become one of the popular methods to prepare surfaces bearing with $\mathrm{OH}$ bonds for coupling reactions that have can be applied in diverse areas. The rapid formation of covalent bonds between the anchoring group and the substrates make this method preferable compared to other conventional methods [1-3]. The stabilization of the monolayer of products is achieved by these covalent bonds allowing ease of additional chemical modification while maintaining the integrity of the newly formed surface film [4]. Moreover, the types, amounts and different conditions of surface grafting largely influences

*Corresponding author : nurul.husna_g03613@utp.edu.my 
the morphology, mechanical, processability and the barrier properties of the nanocomposite [5].

3-aminopropyltiethoxysilane (APTES) is one of a popular choice of coupling agent as it has terminal amines that allow further attachment of molecules and exhibits self-assembly. To attach APTES onto metal oxide surfaces, salinization is often used. This process involves the hydroxylation of silicon dioxide, followed by the hydrolysis of the APTES ethoxy groups with ethanol as the leaving group to produce an aminopropyl-terminated surface [6]. The basic character of the amino groups of APTES enables it to autocatalyze its own condensation and hydrolysis with the absence of additional catalyst during the functionalization process. However, the conformation, density and structure of the covalently attached layer of APTES is strongly affected by the solvent choice and curing methods ${ }^{6}$. In addition, the increase of reaction time can improve the average density of the APTES coverage. To obtain the right structure and position of the terminal amines to be oriented away from the underlying substrate, selecting the right solvent, reaction time and method during functionalization is crucial [7].

The aim of this study is to analyze the influence of various conditions of $\mathrm{ZnO}$ NPs functionalization with APTES on the barrier properties of graphene oxide-zinc oxide (GO$\mathrm{ZnO}$ ) in epoxy matrix. Graphene oxide is known for its impermeability and stable properties, while having extreme van der waal forces between its individual sheets [8]. Anchoring functionalized $\mathrm{ZnO}(\mathrm{f}-\mathrm{ZnO})$ onto graphene sheets is believed to reduce the forces between the sheets, thus improving the barrier properties of epoxy coating when function as fillers. Two different conditions were used to graft APTES onto the ZnO NPs surface. The resulting product is analyzed with FTIR and XPS. The barrier properties of GO-ZnO in epoxy from each type of functionalized $\mathrm{ZnO}$ is also studied using Electrochemical Impedance Test (EIS).

\section{Experimental}

\subsection{Materials}

Zinc oxide NPs were purchased from Aldrich and have a particle size of 50nm. All the materials for functionalizing $\mathrm{ZnO}$ NPs such as 3-aminopropyltiethoxysilane (APTES), toluene and DMSO were purchased from R\&M and used without further purification. Commercial grade graphene oxide sheets dispersed in water $(4 \mathrm{mg} / \mathrm{ml})$ was provided by Graphenea. DMF and acetone was purchased from Merck and was used without further purification. The epoxy resin was a standard diglycidyl ether of bisphenol-A (DGEBA) type EPON 828. The curing agent was polyamide Epikure F205 supplied by ASA chem.

\subsection{Functionalization of ZnO NPs}

\subsubsection{APTES Modification of ZnO using reflux method}

$\mathrm{ZnO}$ dispersion was prepared by mixing $10 \mathrm{~g} \mathrm{ZnO}$ powder and $50 \mathrm{~g}$ toluene in a round bottomed flask by stirring at room temperature using a magnetic stirrer for 5 minutes. The suspension was then treated in an ultrasonic bath for $10 \mathrm{~min} .1 \mathrm{~g}(0.0055 \mathrm{~mole})$ of APTES was added into the suspension and it was stirred at room temperature. After a yellowtransparent dispersion was obtained, the reaction mixtures were then refluxed for a further $24 \mathrm{~h}$. The solvents in the dispersion were evaporated to dryness by using a rotation evaporator. After that, APTES modified $\mathrm{ZnO}$ powder was washed to remove unreacted APTES molecules with ethanol (three times). The powder was dried at $50{ }^{\circ} \mathrm{C}$ in the vacuum oven for 2 hours. The product was denoted as Reflux-Func-ZnO. 


\subsubsection{APTES Modification of ZnO using ultrasonic method}

Briefly, $0.5 \mathrm{~g}$ of $\mathrm{ZnO}$ NPs were dissolved in $10 \mathrm{~mL}$ of DMSO via ultrasonication technique for $1 \mathrm{~h}$ and 30 minutes to form a homogeneous suspension. Then, $10 \mathrm{~mL}$ of APTES was added to the solution and ultrasonicated for 2 hours. The product is removed from the solution using centrifugation technique for $4000 \mathrm{rpm}$ with addition of absolute ethanol. The settled product was collected from the centrifuge tube and dried in an oven at $60^{\circ} \mathrm{C}$ for $24 \mathrm{~h}$ to obtain APTES functionalized ZnO NPs. The product was labelled as Ultra-Func-ZnO.

\subsection{Preparation of Composite Coatings}

The dried GO-ZnO hybrids $(0.012 \mathrm{~g})$ were dispersed in $100 \mathrm{ml}$ of acetone via ultrasonication bath for 1 hour. Once the suspension is homogeneously dispersed, polyamide hardener was added. The mixture was further ultrasonicated at $60^{\circ} \mathrm{C}$ for 3 hours to allow for slow solvent evaporation while maintaining the homogeneous distribution of GO-ZnO hybrids. An additional 2 hours of heating in the oven was carried out to ensure complete removal of acetone. The epoxy resin was then added to the suspension according to the weight ratio of 100:58 and mixed for 5 minutes. The resulting mixture were sprayed onto the steel surface by a gravity spray gun. The sprayed steel surface was post cured for 24 hours at room temperature and post cured in the vacuum oven at $100^{\circ} \mathrm{C}$ for 2 hours.

\subsection{Characterization}

X-ray photoelectron spectroscopy (XPS) testing was carried out on a Thermoscientific kalpha system. The pass energy of X-ray microprobe was selected at $200 \mathrm{eV}$. FTIR spectra were recorded in transmission mode on $\mathrm{KBr}$ pallets using FT/IR-430 spectrometer over wavelength range of 500-4000 $\mathrm{cm}^{-1}$. The quality of the dispersion of fillers in epoxy composite was observed by field emission scanning electron microscopy (FESEM). The barrier properties of the nanocomposites were evaluated by electrochemical impedence spectroscopy (EIS) to observe the electrochemical behavior of the coated substrate. Three electrode cell configurations were used to take the measurement, where the substrate served as the working electrode, graphite rod as the counter electrode and the silver/silver chloride $(\mathrm{Ag} / \mathrm{AgCl})$ as the reference electrode. The corrosive electrolyte for all the electrochemical evaluation tests was $3.5 \mathrm{wt} \% \mathrm{NaCl}$ solution. The open circuit potential (OCP) were recorded for all data followed by EIS tests in the frequency range of $10^{-2}$ to $10^{5} \mathrm{~Hz}$ by utilizing $10 \mathrm{mV}$ AC amplitude after immersion in corrosive electrolyte for 7 days. The obtained electrochemical data was analyzed using Nova 1.10 software.

\section{Results and discussion}

\subsection{Characterization of APTES-Functionalized ZnO}

\subsubsection{FTIR Analysis}

FTIR spectra of the APTES-modified and the non-modified $\mathrm{ZnO}$ nanoparticles are shown in Fig 1. FTIR spectrum of APTES functionalized $\mathrm{ZnO}$ (both Ultra-Func-ZnO and RefluxFunc-ZnO) reveals the addition of $\mathrm{NH}_{2}$ stretch vibrations at $2930 \mathrm{~cm}^{-1}$ as compared to unmodified $\mathrm{ZnO}$. The $\mathrm{Zn}-\mathrm{O}-\mathrm{Si}$ bending vibration at $970 \mathrm{~cm}^{-1}$ confirms the successful attachment of APTES on the surface of zinc oxide. Further, the overlap and appearance of $\mathrm{Si}-\mathrm{H}$ and amine vibration bands at $778 \mathrm{~cm}^{-1}$ also confirm the chemical functionalization of 
$\mathrm{ZnO}$ by APTES. The stretching vibrations at $1364 \mathrm{~cm}^{-1}$ is assigned to the $\mathrm{C}-\mathrm{N}$ bonding and the asymmetric peak at $1166 \mathrm{~cm}^{-1}$ indicates the $-\mathrm{Si}-\mathrm{O}$ bonding. These FTIR results affirmed the attachment of APTES onto the surface of the ZnO NPs.

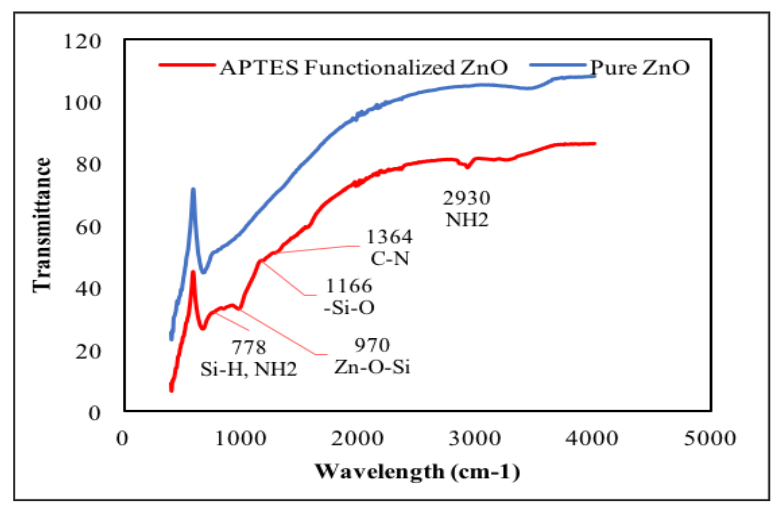

Fig 1. FTIR spectrum of APTES-Functionalized $\mathrm{ZnO}$ and pure $\mathrm{ZnO}$

\subsubsection{XPS Analysis}

XPS analysis was conducted to monitor the attachment of APTES layer onto $\mathrm{ZnO}$ by reflux and ultrasonication method. In general, the anchoring mechanism of organosilane molecules can be classified into two processes: Condensation and hydrogen bonding [4]. Condensation will result in formation of siloxane bonds that is preferable for APTES functionalization process [6]. The amines terminal groups are pointing outwards, ready for further attachment with graphene oxide sheets. Meanwhile, hydrogen bonding of amine groups with the surface of the metal oxide would expose the ethoxy group to point away from the substrate, preventing further functionalization. The configuration of APTES on the surface of ZnO NPs can be confirmed by the N1s spectrum (Figure 1 and 2) showing peaks attributable to different attachment modes of APTES.

Siloxane bonds are formed at $402.4 \mathrm{Ev}$ while hydrogen bonds can be assigned to peak 400.9 $\mathrm{eV}$ [9]. Both bonds can be identified in Fig 2 and Fig 3. However, the intensity of peaks differs from each ZnO NPs samples. The appearance of a higher peak intensity of silane coupling for Reflux-Func-ZnO indicates that more siloxane bonds are formed as compared to hydrogen bonding of amine groups onto the substrates (reverse amino coupling). It is expected that the increased reaction time during the reflux process allowed the APTES to form strong siloxane bonds on the metal oxide surface. In this case, ultrasonication alone is insufficient to stimulate condensation reaction resulting in an insignificant atomic percentage of siloxane bonds formed on the Ultra-Func-ZnO surface (Table 1).

Table 1. Atomic percentage of bonding for different APTES Functionalizing method

\begin{tabular}{|c|c|c|}
\hline Mechanism/Atomic \% & Reflux-Func-ZnO & Ultra-Func-ZnO \\
\hline Siloxane bonding & $32.3 \%$ & $7.8 \%$ \\
\hline Hydrogen bonding & $67.7 \%$ & $92.2 \%$ \\
\hline
\end{tabular}




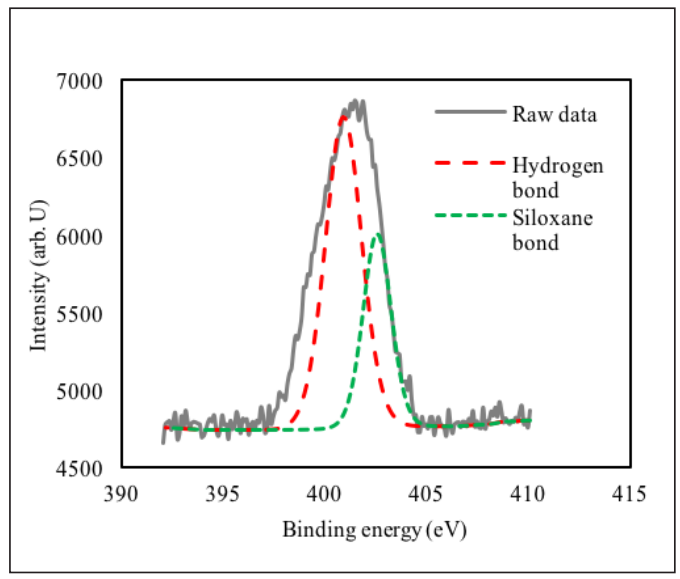

Fig 2. High-resolution XP spectra from Reflux-Func-ZnO

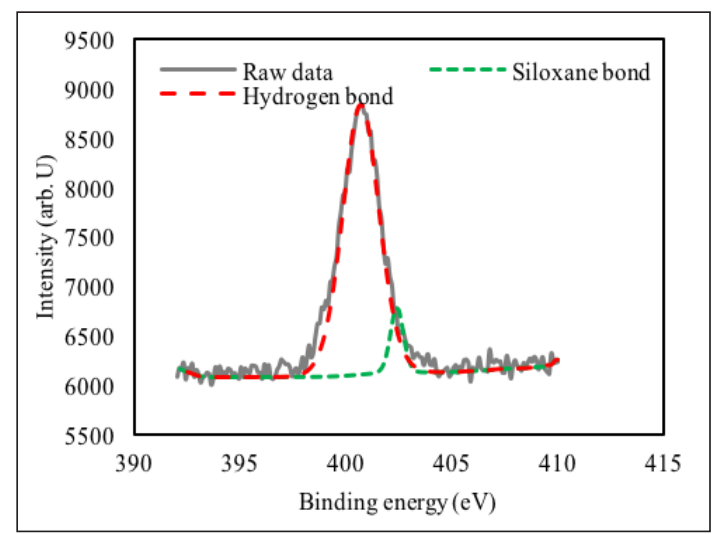

Fig 3. High-resolution XP spectra from Ultra-Func-ZnO

\subsection{Characterization and Barrier properties of Epoxy containing GO-ZnO Nanocomposite Coating}

\subsubsection{Characterization of GO-ZnO nanocomposite coating}

To confirm the successful attachment of both Reflux and Ultra functionalized $\mathrm{ZnO}$ onto the graphene oxide sheets, FTIR was conducted for GO-ZnO nanocomposites. There is an intensive broaden peak at around $3200 \mathrm{~cm}^{-1}$ for Graphene Oxide (GO) conveying the intercalated structure of $\mathrm{C}-\mathrm{H}$ and water molecules in graphene oxide sheets. The similar type of peak was identified in samples GO-ZnO proving that the graphene sheet-like structure is retained after modification with APTES-Functionalized $\mathrm{ZnO}$. A good interaction between $\mathrm{ZnO}$ and $\mathrm{GO}$ is observed with the presence of $\mathrm{ZnO}$ at peak $662 \mathrm{~cm}^{-1}$ in GO-ZnO nanocomposite. However, the intensity at $1644 \mathrm{~cm}^{-1}(\mathrm{C}=\mathrm{O}$ groups $)$ become weak in the FTIR spectrum. 


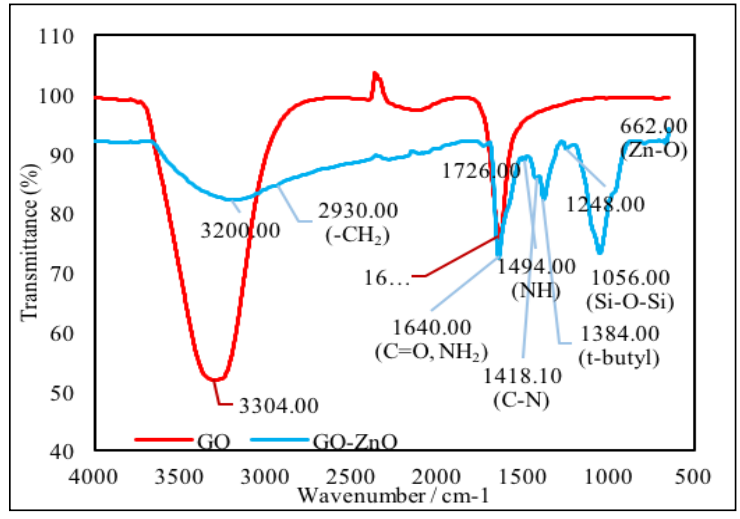

Fig 4. FTIR spectrum of $\mathrm{GO}$ and $\mathrm{GO}-\mathrm{ZnO}$ nanocomposite coating

\subsubsection{Barrier Properties of GO-ZnO nanocomposite coating}

The effect of different methods of ZnO NP APTES functionalizing on the corrosion and ionic resistances of epoxy coating was studied by EIS technique. EIS was performed at 7 days of immersion in $3.5 \mathrm{wt} \% \mathrm{NaCl}$ solution. The Nyquist and bode plot of $\mathrm{GO}-\mathrm{ZnO}$ produced by Reflux-func- $\mathrm{ZnO}$ and ultra-func- $\mathrm{ZnO}$ after 7 days of immersion is demonstrated in Figure 5. At a wide frequency range, the coating was mostly resistive, and the capacitive behaviors can be detected at very high frequencies. The Nyquist plot clearly showed the existence of onetime constant semicircle after 7 days of immersion for all the samples tested. At this stage, oxygen, water and corrosive ions such as $\mathrm{Cl}^{-}$has penetrated the coating through the inherent micro-pores and has not yet reached the surface of the steel $[10,11]$. Different APTES functionalization method of $\mathrm{ZnO}$ NPs have significant impact on its barrier properties. The radius of the impedance arc was the largest for GO- $\mathrm{ZnO}$ prepared by reflux-Func-ZnO than $\mathrm{GO}-\mathrm{ZnO}$ prepared by ultra-func- $\mathrm{ZnO}$ and $\mathrm{EP}$. In addition, the capacitive region and the maximum phase angle of GO-ZnO prepared by reflux-Func-ZnO are at a wider frequency region after 7 days of immersion. To further understand the inhibition mechanism of the nanocomposite coating, the EIS data was fitted to an equivalent circuit using NOVA 1.10 software. The proposed equivalent circuit for the coated steel substrate after 7 days of immersion in $3.5 \mathrm{wt} \% \mathrm{NaCl}$ solution is shown in Figure 6. The circuit consists of solution resistance $\left(R_{s}\right)$, charge transfer resistance $\left(R_{c t}\right)$ and constant phase element of double layer $\mathrm{CPE}_{\mathrm{dl}}$. The values of the impedance parameters extracted from the equivalent models are given in Table 2. To evaluate the improvement of the method of functionalization of $\mathrm{ZnO}$ on the corrosion properties of GO-ZnO nanocomposite, the protection efficiency was calculated based on the Rct parameter as indicated in the equation below [12]:

$$
\eta=\frac{R_{c t}(c)-R_{c t}(e p)}{R_{c t}(c)} \times 100
$$

where $R_{c t}(c)$ is the charge transfer resistance of GO-ZnO coating and $R_{c t}(e p)$ as the charge transfer resistance of bare epoxy. The protection efficiency of GO-ZnO prepared by Reflux-func- $\mathrm{ZnO}$ and ultra-func- $\mathrm{ZnO}$ is $97 \%$ and $94 \%$ respectively. It is clear from the results that incorporating GO-ZnO prepared by Reflux-func- $\mathrm{ZnO}$ and ultra-func- $\mathrm{ZnO}$ into the epoxy coating caused a significant rise of Rct compared to EP. Considerable enhancement of protection efficiency can be observed for samples prepared by Reflux-func-ZnO, indicating that the large number of siloxane bonds formed at the $\mathrm{ZnO}$ nanoparticle surface managed to 
expand the GO sheets and thus improve the dispersion of fillers in the epoxy matrix, delivering excellent barrier properties.
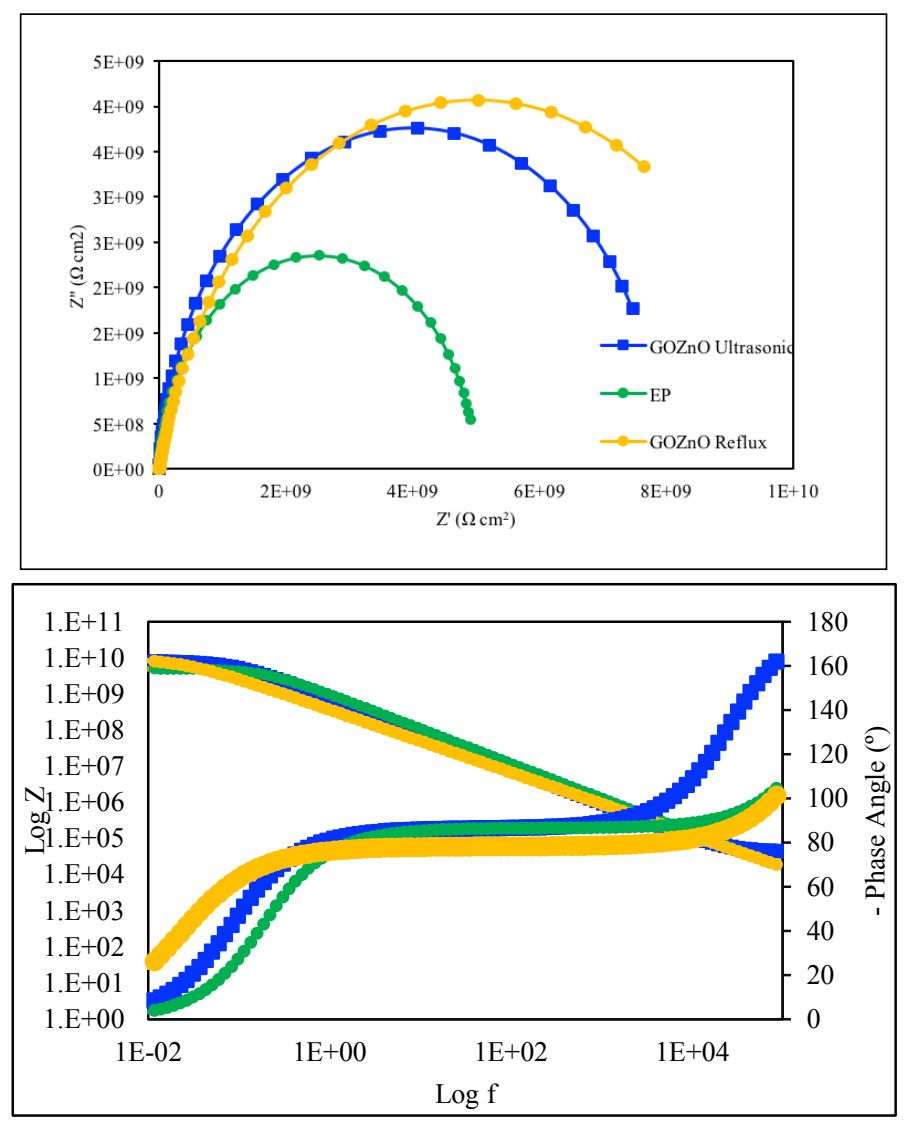

Fig 5. Nyquist and bode plot for EP, EP contained GO-ZnO from Reflux-Func-ZnO and EP contained $\mathrm{GO}-\mathrm{ZnO}$ from ultra-Func-ZnO

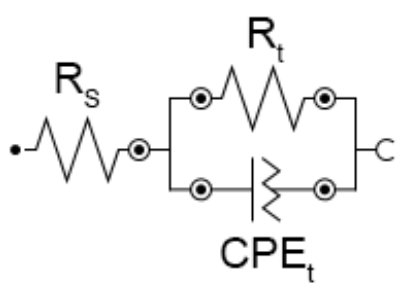

Fig 6. Equivalent circuit of the fitted nyquist and bode plots for all samples 
Table 2. Equivalent circuit values for EP, EP contained GO-ZnO from Reflux-Func- $\mathrm{ZnO}$ and EP contained $\mathrm{GO}-\mathrm{ZnO}$ from ultra-Func-ZnO

\begin{tabular}{|l|l|l|l|}
\hline Sample & \multirow{2}{*}{$\mathbf{R}_{\mathbf{c t}}\left(\mathbf{G} \boldsymbol{\Omega} \mathbf{c m}^{\mathbf{2}}\right)$} & $\mathbf{C P E _ { \mathbf { d l } }}$ \\
\cline { 2 - 4 } & & $\mathbf{Y}_{\mathbf{0}}\left(\mathbf{\Omega}^{-\mathbf{1}} \mathbf{c m}^{-\mathbf{2}} \mathbf{s}^{\mathbf{n}}\right)$ & $\mathbf{n}$ \\
\hline Blank EP & 0.5 & $1.85 \times 10^{-10}$ & 0.952 \\
\hline $\begin{array}{l}\text { GO-ZnO by Reflux- } \\
\text { Func-ZnO }\end{array}$ & 9.5 & $4.9 \times 10^{-10}$ & 0.87 \\
\hline $\begin{array}{l}\text { GO-ZnO by Ultra- } \\
\text { Func-ZnO }\end{array}$ & 8 & $2.64 \times 10^{-10}$ & 0.89 \\
\hline
\end{tabular}

\section{Conclusions}

The effect of different methods of silanization reaction with APTES of ZnO NPs on the corrosion protection of steel has been investigated. The reaction time during the silanization plays an important role in making sure that the configuration of amine terminals is accurate for further attachment with graphene oxide sheets. The increment of $3 \%$ of the protection efficiency was observed for $\mathrm{GO}-\mathrm{ZnO}$ prepared by Reflux-func- $\mathrm{ZnO}$ as compared to $\mathrm{GO}-\mathrm{ZnO}$ prepared by ultra-func-ZnO. This is because reflux method allowed the condensation of siloxane bonds on the surface of the metal oxide to proceed, which subsequently affected the barrier properties of the produced $\mathrm{GO}-\mathrm{ZnO}$ by improving the dispersion of graphene oxide sheets in the matrix. This can be demonstrated with the results obtained from FTIR, XPS and electrochemical studies.

\section{References}

1. H. Wang, Z. Liu, E. Wang, X. Zhang, R. Yuan, S. Wu, and Y. Zhu, Appl. Surf. Sci., 357, pp. 229-235, (2015).

2. M. F. Montemor, Surf. Coatings Technol., 258, pp. 17-37, (2014).

3. B. Ramezanzadeh, E. Ghasemi, M. Mahdavian, E. Changizi, and M. H. Mohamadzadeh Moghadam, Carbon N. Y., 93, pp. 555-573, (2015).

4. A. F. Jaramillo, R. Baez-cruz, L. F. Montoya, C. Medinam, E. Pérez-tijerina, and F. Salazar, Ceram. Int., 43, no. 15, pp. 11838-11847, (2017).

5. K. K. Jena, R. Narayan, and K. V. S. N. Raju, Prog. Org. Coatings, 89, pp. 82-90, (2015).

6. R. G. Acres, A. V Ellis, J. Alvino, C. E. Lenahan, D. A. Khodakov, G. F. Metha, and G. G. Andersson, J. Physical Chem.(2012).

7. N. N. Rabin, J. Morshed, H. Akhter, S. Islam, A. Hossain, M. Alam, M. R. Karim, and M. A. Hasnat, Int. J. Chem. React. Eng. pp. 1-10, (2015).

8. Y. Cui, S. I. Kundalwal, and S. Kumar, Carbon N. Y., 98, pp. 313-333, (2016).

9. F. Grasset, N. Saito, D. Li, D. Park, I. Sakaguchi, N. Ohashi, H. Haneda, T. Roisnel, S. Mornet, and E. Duguet, J. of Alloys \& Compounds, 360, pp. 298-311, (2003).

10. Z. Yu, H. Di, Y. Ma, Y. He, L. Liang, L. Lv, X. Ran, Y. Pan, and Z. Luo, Surf. Coatings Technol., 276, pp. 471-478, (2015).

11. B. Ramezanzadeh, S. Niroumandrad, A. Ahmadi, M. Mahdavian, and M. H. Mohamadzadeh Moghadam, Corros. Sci., 103, pp. 283-304, (2016).

12. N. Harfouche, N. Gospodinova, B. Nessark, and F. X. Perrin, J. Electroanal. Chem., 786, pp. 135-144, (2017). 